\title{
Journal of Surgery
}

\section{A New Cavoplasty in Domino Liver Transplantation for Familial Amyloidotic Polyneuropathy Using Arterial Donor Graft}

\section{Eduardo de Souza Martins Fernandes ${ }^{1,2}$, Leandro Savattone Pimentel ${ }^{2}$, Felipe Pedreira Tavares de Mello ${ }^{2}$, Joaquim Ribeiro Filho $^{1}$, Camila Liberato Girão ${ }^{2}$, Ronaldo Oliveira Andrade², Camilla César ${ }^{2}$}

${ }^{1}$ Department of Surgery - Rio de Janeiro Federal University-RJ

${ }^{2}$ Department of Abdominal Surgery and Transplantation - Hospital Adventista Silvestre- RJ

"Corresponding author: Eduardo de Souza Martins Fernandes, Department of Surgery and Liver Transplant, Hospital Adventista Silvestre, Rio de Janeiro, Brazil. Email: esmfernandes@hotmail.com

Citation: Fernandes ESM (2018) A New Cavoplasty in Domino Liver Transplantation for Familial Amyloidotic Polyneuropathy Using Arterial Donor Graft. J Surg: JSUR-1122. DOI: 10.29011/2575-9760. 001122

Received Date: 23 March, 2018; Accepted Date: 31 March, 2018; Published Date: 09 April, 2018

\begin{abstract}
Since the domino transplantation was first performed several techniques were introduced for outflow reconstruction. An innovative discovery was used in nine successful cases that are described from the authors. In this description it is proposed using iliac artery by performing a neo-suprahepatic cava facilitating the anastomosis
\end{abstract}

\section{Introduction}

In 1997 a new concept of liver transplantation, "the domino liver transplantation" was introduced [1]. In the original description a graft from a carrier of familial amyloidotic polyneuropathy (FAP) was used as "domino donor" for a compatible recipient, increasing the pool of hepatic grafts was used as "domino donor was accepted worldwide; a web-based registry for those patients was created and international experience has been reported [2].

The best technique for caval anastomosis in domino transplantation recipients is not well defined. We devised a new technique for outflow reconstruction that was adopted in cases of domino transplantation. The outflow reconstructions are a very important technical part of this procedure and many different venous grafts have been used to perform the double piggy back technique as described by Dr Barroso in Portugal. A venoplasty between the stumps of the amyloidotic graft was performed whenever possible using the iliac veins or caval renal junction [3] (Figures 1,2).

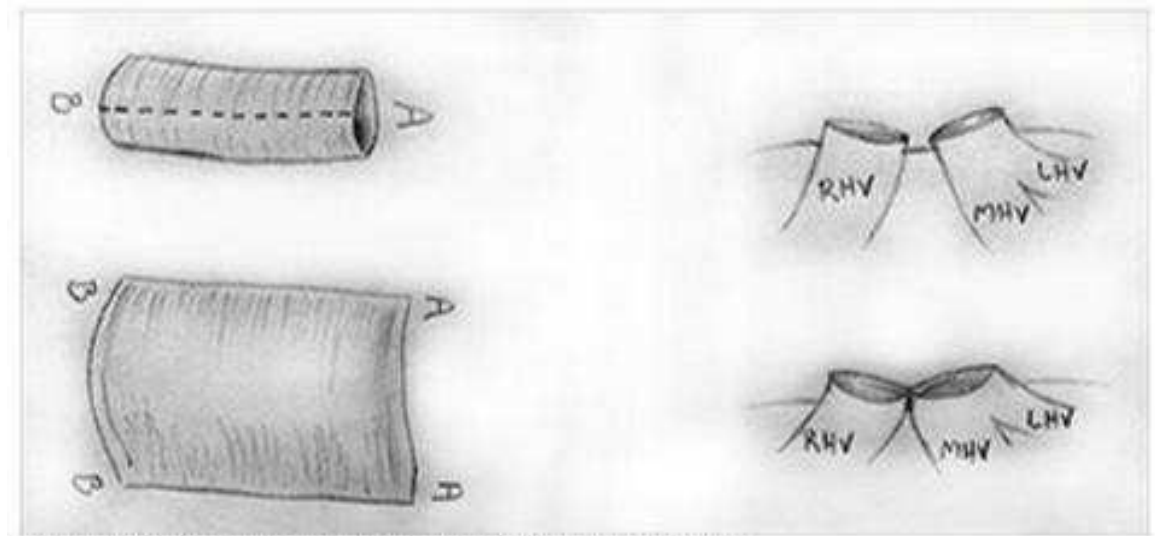

Cadaveric iliac artery graft is longitudinally opened /

The ostiumof the right hepatic vein is joined to the middle and left hepatic veins

Figure 1: Cadaveric iliac artery graft is longitudinally opened/ The ostium of the right hepatic vein is joined to the middle and left hepatic veins. 


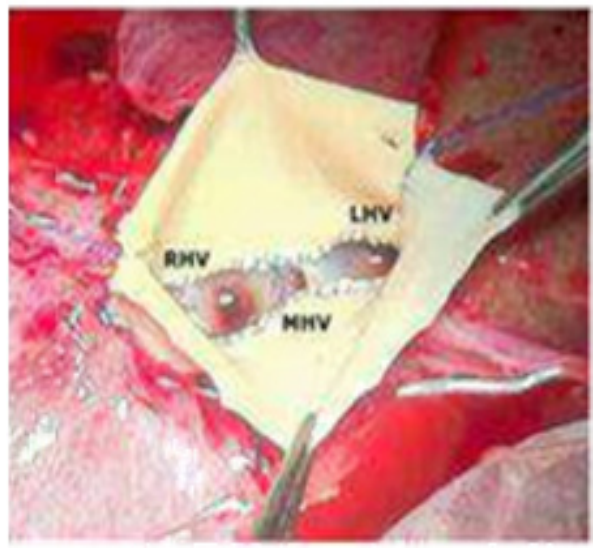

Figure 2a: The ostium of the right hepatic vein is joined to the media and left hepatic veins.

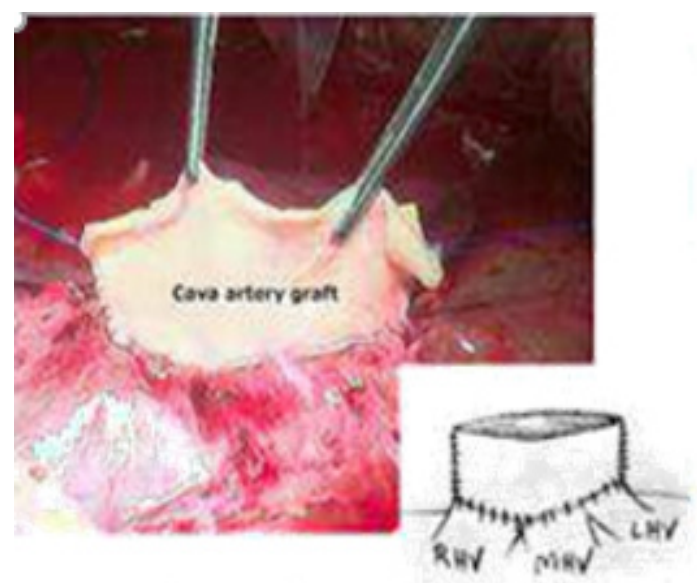

Figure 2b: Completed reconstruction of venous outflow

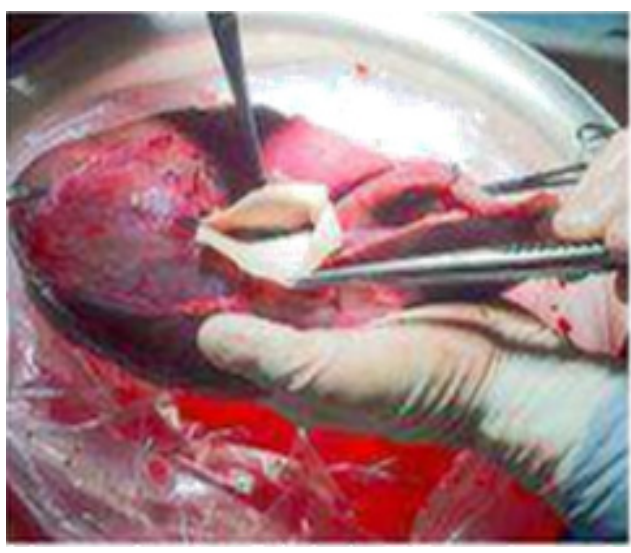

Figure 2c: Final aspect of the graft.

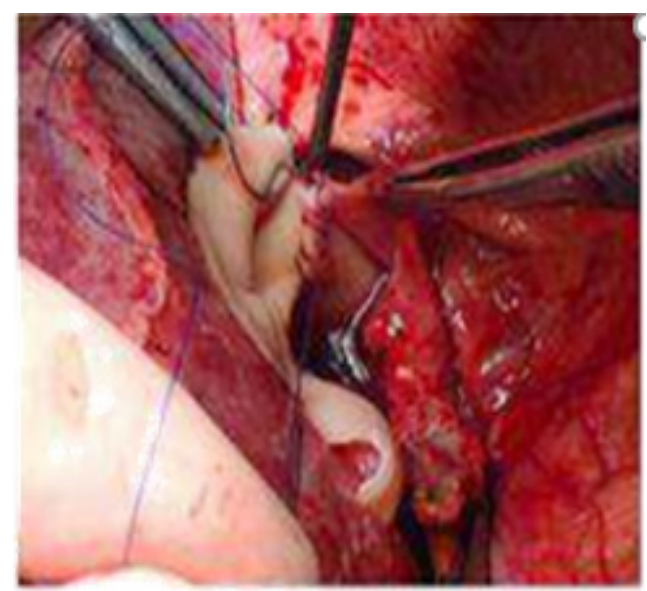

Figure 2d: Caval anastomosis.

of venous liver with cadaveric iliac artery graft.

\section{Methods}

A retrospective study of nine cases of domino transplantation with the use of arterial patch to reconstruct the hepatic veins during the bench procedure. The left and middle veins are keep together and then united with the right hepatic vein - venoplasty with a running suture of 5-0 polypropylene. After that this single cuff is surrounded with an arterial iliac donor artery to expand the venous outflow and facilitate the caval anastomosis. In all cases the orifices of major hepatic veins of the amyloidotic livers did not have sufficient tissue to perform a direct anastomosis with the caval cuff of the domino transplantation recipients, in whom a piggyback reconstruction has been planned.

\section{Discussion}

The original technique used for FAP liver transplantation has been modified to encourage the use of domino sequential transplant as another source of liver grafts.

Vena cava reconstruction is a determinant part of graft function in liver transplantation in all kind of graft and the adequate outflow is expected for the success of all parenquima circulation. In domino transplantation several types of venous grafts have been suggested to reconstruct the short veins left in the fap liver. In the present description we propose a new technique that differs from the technique mentioned above. We used a neo-suprahepatic cava, constructed using iliac artery graft, facilitating the anastomosis as if was a regular cadaveric liver transplant. The venous grafts are usually very tiny and could be a potential problem in case of disrupter and bleeding after reperfusion [4-8].

The arterial iliac vessel hasn't been described for this proposes and our initial experience demonstrated that the anastomosis is safer and easier. 


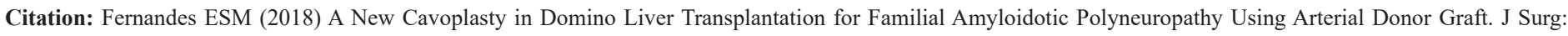
JSUR-1122. DOI: 10.29011/2575-9760. 001122

\section{Conclusion}

Although this series is small, our results suggest that hepatic venous outflow reconstruction using iliac artery from deceased donor graft can be one way to ensure a safe double piggyback domino liver transplantation. Arterial iliac grafts haven't been published to increase the safeness of domino and provide excellent outflow patency.

\section{References}

1. Furtado A, Tome L, Oliveira FJ, Furtado E, Viana J, et al. (1997) Sequential liver transplantation. Transplant Proc 29: 467.

2. The Familial Amyloidotic Polyneuropathy World Transplant Registry: The Domino Liver Transplant Registry.

3. Liu C, Loong C, Hsia C, Tsou MY, Tsai HL, et al. (2008) Venoplasty of hepatic venous outflow with a venous patch in domino liver transplantation. Liver Transpl 14: 1378.
4. Pacheco-Moreira LF, Oliveira M, Balbi E, da Silva AC, Miecznikowski $R$, et al. (2003) A new tecnical option for domino liver transplantation. Liver Transpl 9: 632.

5. Cerqueira A, Pacheco-Moreira L, Enne M, Alves J, Amil R, et al (2006) Outflow reconstruction in domino liver transplantation with interposition of autologus portal vein graft. A new technical option in living donor domino liver transplant scenario. Liver Transpl 12: 1298.

6. Cescon M, Grazi GL, Ravaioli M, Cucchetti A, Ercolani G, et al. (2007) Modified outflow reconstruction with a venous patch in domino liver transplantation. Liver Transpl 13: 1756-1757.

7. Jabbour N, Gagandeep S, Genyk Y, Selby R, Mateo R (2006) Caval preservation with reconstruction of the hepatic veins using cavalcommon iliac bifurcation graft for domino liver transplantation. Liver Transpl 12: 324-325.

8. Tzakis AG (2000) Let's play dominos. Liver Transpl 6: 506-508. 